\title{
BMJ Open Patient-reported outcomes (PROs) in randomised controlled trials in diabetes and pregnancy: protocol for a systematic review
}

Christine Newman (D) , ${ }^{1}$ Oratile Kgosidialwa, ${ }^{1}$ Louise Dervan, ${ }^{1}$ Delia Bogdanet (D) ${ }^{2}$ Aoife Maria Egan (D) , ${ }^{3}$ Linda Biesty, ${ }^{4}$ Declan Devane, ${ }^{4,5,6,7,8}$ Paula M O'Shea, ${ }^{9}$ Fidelma Dunne ${ }^{1}$

To cite: Newman C, Kgosidialwa 0, Dervan L, et al. Patient-reported outcomes (PROs) in randomised controlled trials in diabetes and pregnancy: protocol for a systematic review. BMJ Open 2021;11:e052506. doi:10.1136/ bmjopen-2021-052506

- Prepublication history for this paper is available online. To view these files, please visit the journal online (http://dx.doi. org/10.1136/bmjopen-2021 052506).

Received 19 April 2021 Accepted 14 October 2021
Check for updates

(C) Author(s) (or their employer(s)) 2021. Re-use permitted under CC BY-NC. No commercial re-use. See rights and permissions. Published by BMJ.

For numbered affiliations see end of article.

Correspondence to Dr Christine Newman; christine.newman@nuigalway.ie

\section{ABSTRACT}

Introduction Diabetes mellitus is the most common metabolic complication of pregnancy and its prevalence worldwide is rising. The number of randomised controlled trials (RCTs) being conducted in people with diabetes is also increasing. Many studies preferentially publish findings on clinical endpoints and do not report patientreported outcomes (PROs). In studies that do include PROs, PRO reporting is often of poor quality.

Methods We will conduct this systematic review following the Preferred Reporting Items for Systematic Reviews and Meta-Analyses guidelines. Using a combination of medical subject headings and keywords (combined using Boolean operators), we will search webbased databases (PubMed, Cochrane and EMBASE) for RCTs published in English between 2013 and 2021. Two reviewers will review titles and abstracts. We will review the full texts of any relevant abstracts and extract the following data: date of publication or recruitment period, journal of publication, country of study, multicentre or single centre, population and number of participants, type of intervention, frequency of PRO assessment and type of PRO (or PRO measurement) used. We will also record if the PRO was a primary, secondary or exploratory outcome. We will exclude reviews, observational studies, unpublished data for example, conference abstracts and trial protocols. Any published RCT that includes data on a PRO as a primary or secondary outcome will then be compared against the Consolidated Standards of Reporting Trials-Patient-Reported Outcome extension checklist, a structured and approved framework for the publication of results of PROs.

Ethics and dissemination Ethical approval to conduct this study was obtained from the ethics committee at Galway University Hospitals on 24 March 2021 (CA 2592). We aim to publish our findings in a peer-reviewed journal and present our findings at national and international conferences.

Systematic review registration This systematic review was registered prospectively with the International Prospective Register of Systematic Reviews (PROSPER0). Registration number CRD42021234917.

\section{Strengths and limitations of this study}

- This is the first systematic review of patientreported outcomes (PROs) in the area of diabetes in pregnancy.

- It will describe the adequacy of reporting in randomised controlled trials including PROs as a primary or secondary outcome.

- This systematic review is limited to trials conducted during pregnancy only and does not evaluate studies that aim to prevent the development of type 2 diabetes in women with previous gestational diabetes mellitus.

\section{INTRODUCTION}

Diabetes is the most common medical complication of pregnancy and causes considerable maternal and foetal morbidity. ${ }^{1}$

The International Diabetes Federation estimates that $2.2 \%$ of all pregnancies are affected by pregestational diabetes mellitus (PGDM) and it is well established that women with PGDM and their offspring have increased rates of complications including macrosomia, congenital anomaly, Caesarean section, pre-eclampsia and stillbirth. ${ }^{2-8}$

Gestational diabetes mellitus (GDM) is defined as any degree of glucose intolerance with onset or first recognition during pregnancy and usually resolves after the birth of the placenta. The prevalence of GDM is increasing worldwide paralleling the increasing rates of obesity and rising maternal age. Follow-on data from the landmark Hyperglycaemia and Adverse Pregnancy Outcomes study ${ }^{9}$ indicate that the worldwide prevalence of GDM is between $9.3 \%$ and $25.5 \% .^{10}$

Assessing the frequency and burden of GDM is not straightforward. The prevalence of GDM is affected by the use of different diagnostic criteria, rates of obesity and ethnic 
variations. $^{211-13}$ Data regarding the prevalence of GDM are also affected by the use of universal versus risk-factor based screening; studies from two different centres in the Ireland in the last 10 years identified rates of GDM varying from $27.4 \%$ in those with identifiable risk factors ${ }^{14}$ versus $12.4 \%$ when universal screening is employed. ${ }^{11}$

As with PGDM, GDM is associated with complications for both mother and infant. Short-term complications include large for gestational age infants and macrosomia $^{15}$ and hypertensive disorders ${ }^{16}$; and long-term complications include increased risk of obesity and glucose intolerance for both offspring ${ }^{17}$ and mother. ${ }^{18}$ Obesity alone without dysglycaemia is also associated with adverse pregnancy outcomes ${ }^{19}$ and there is growing evidence to support the additive risks posed by a combination of diabetes and obesity. ${ }^{20}$

Outside of pregnancy, the management and complications of diabetes can cause substantial distress for patients. ${ }^{21}$ This stress can be exacerbated by pregnancy and many women report anxiety during pregnancy. ${ }^{22}$ Furthermore it is well recognised that diabetes distress in pregnancy is a poor prognostic indicator and correlates with poor pregnancy outcomes. ${ }^{23}$

In recent years there has been a large increase in the number of randomised controlled trials (RCTs conducted in pregnancy; these studies often publish information on physiological parameters and frequently exclude psychological or quality of life data. Two recent core outcome sets (COSs) published in the areas of diabetes in pregnancy provide evidence that stakeholders often prioritise information on physiological, biochemical and birth outcomes over psychological and quality of life outcomes, even when such study groups include patient advocates and representative. ${ }^{24}{ }^{25}$ While COSs provide expert guidance for researchers, these parameters fail to capture the unseen burden of therapeutic interventions. ${ }^{26}$

Patient-reported outcomes (PROs) are defined as 'any report of the status of a patient's health condition that comes directly from the patient without interpretation of the patient's response by a clinician or anyone else'. ${ }^{27}$ PROs allow healthcare providers to appreciate 'patients' perspectives regarding treatment benefit and harm, directly measure treatment benefit and harm beyond survival, major morbid events and biomarkers, and are often the outcomes of most importance to patients and families'. ${ }^{27}$

PROs are increasingly incorporated into clinical trials to conduct cost analyses ${ }^{28}$; however, their use is not limited to this and some patient-reported-outcome measures (PROMs) correlate better with biochemical markers of disease activity or severity than traditional symptoms of the disease. ${ }^{29}$

The importance and value of PROs is well established in other areas of medicine however searches of webbased registries (PubMed, Cochrane, EMBASE, World of Science and CINAHL) identified no data on the use of PRO in studies of diabetes in pregnancy and a review of PROSPERO (a web-based registry for systematic reviews)
Table 1 PICO statement for this systematic review

$\begin{array}{ll}\text { Population } & \begin{array}{l}\text { Pregnant women with diabetes in pregnancy } \\ \text { (either pregestational or gestational) }\end{array} \\ \text { Intervention } & \begin{array}{l}\text { Any intervention during pregnancy, regardless } \\ \text { of the duration of the intervention. Such } \\ \text { interventions include but are not limited to the } \\ \text { use of insulin or oral hypoglycaemic agents; } \\ \text { insulin delivery systems; glucose monitoring } \\ \text { of any description-frequency/continuous } \\ \text { glucose monitoring, flash glucose monitoring; } \\ \text { educational interventions; mHealth or mobile } \\ \text { phone applications for contact; medications; } \\ \text { exercise; dietary input or dietician input. }\end{array} \\ \text { Comparison } & \text { Any } \\ \text { Outcome } & \text { Any patient-reported outcome }\end{array}$

found no systematic reviews summarising PROMs in this area. Our systematic review of PROs would be the first of its kind conducted in diabetes in pregnancy.

\section{Objectives}

This paper presents a protocol for a systematic review of the use of PROs in diabetes in pregnancy trials. Specifically we will identify the number of RCTs conducted in diabetes in pregnancy that have included a PRO in their primary or secondary endpoints (table 1).

The completeness and quality of reporting in these studies will be evaluated by comparing them to the Consolidated Standards of Reporting Trials-Patient-Reported Outcome (CONSORT-PRO) extension, a framework for grading the quality of PRO reporting in RCTs. ${ }^{30}$

\section{METHODS AND ANALYSIS}

This protocol has been registered and approved within the PROSPERO database (CRD42021234917). This systematic review will be conducted in compliance with the Preferred Reporting Items for Systematic Reviews and Meta-Analyses guidelines.

\section{Search strategy}

We will search PubMed, CENTRAL via the Cochrane library and EMBASE online databases for RCTs published in English between 1/1/2013 and 20/1/2021. The CONSORT-PRO extension framework was published in 2013, so only studies published after 2013 are suitable for evaluation. The databases will be searched using a combination of medical subject headings headings and keywords (combined using Boolean operators). We will also manually search the references of published RCTs fitting the inclusion criteria, to ensure all relevant studies are found. Grey literature including Google Scholar, trial registries and conference abstracts will not be included as we aim to report on the quality of reporting in full-text published articles.

The PICO format will be used to guide the search strategy and is described in table 1 . 


\begin{tabular}{|c|c|c|}
\hline ID & Search & Hits \\
\hline$\# 1$ & $\begin{array}{l}\text { MeSH descriptor: (Pregnancy in } \\
\text { Diabetics) explode all trees }\end{array}$ & 314 \\
\hline \#2 & Diabet $^{\star}$ ti,ab,kw & 95912 \\
\hline \#3 & Gestational diabetes ti;ab;kw & 3348 \\
\hline \#4 & Gestational diabetes mellitus & 2478 \\
\hline \#5 & GDM ti;ab;kw & 1329 \\
\hline \#6 & Pregnan* ti,ab,kw & 53093 \\
\hline$\# 7$ & Hyperglycaemi* ti,ab,kw & 1456 \\
\hline \#8 & Hyperglycemi ti,ab,kw & 8419 \\
\hline \#9 & Glycaemi* ti,ab,kw & 6031 \\
\hline \#10 & 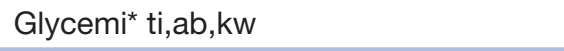 & 15327 \\
\hline \#11 & 1 or 2 or 3 or 4 or 5 or 7 or 8 or 9 or 10 & 102855 \\
\hline \#12 & Antenatal ti,ab,kw & 4697 \\
\hline \#13 & Ante-natal ti,ab,kw & 65 \\
\hline \#14 & Prenatal ti,ab,kw & 7005 \\
\hline \#15 & Pre-natal ti,ab,kw & 63 \\
\hline \#16 & \#6 OR \#12 OE \#13 OR \#14 OR \#15 & 70816 \\
\hline \#17 & \#11 AND \#16 & 11017 \\
\hline
\end{tabular}

GDM, gestational diabetes mellitus; $\mathrm{MeSH}$, medical subject headings.

A sample search strategy is demonstrated in table 2.

\section{Exclusion criteria}

We will exclude studies published before 2013; unpublished data; conference abstracts; trial registries and trial protocols; reviews; observational studies; studies evaluating the effect of prepregnancy or postpregnancy interventions; studies evaluating postnatal interventions which aim to prevent GDM or prevent the progression of GDM to type 2 diabetes; studies which compared different diagnostic criteria for GDM and studies evaluating the effectiveness of interventions for women with obesity, polycystic ovarian syndrome or 'high risk' pregnancies, a subset of whom may have diabetes.

\section{Identifying papers, data extraction and analysis}

All publications identified by the above search strategies will be managed in Endnote X9.

Following an initial scoping review, two reviewers independently reviewed 30 abstracts to ensure their inclusion criteria are aligned and will separately review all titles and abstracts to ensure their suitability $(\mathrm{CN}, \mathrm{LD})$. Any disagreements will be resolved through consultation with a third author (FD).

Full-text papers of selected studies will be reviewed by two reviewers $(\mathrm{CN}, \mathrm{LD})$ who will each independently evaluate the completeness of PRO reporting by comparing published data against the CONSORT-PRO checklist.

One point will be given to each item of the CONSORT-PRO checklist a study meets. This score will

\section{Box 1 Outcomes to be collected from each RCT}

- Quality against Consolidated Standards of Reporting Trials 2010 guidelines.

- Study type (eg, pilot randomised controlled trial (RCT), cluster RCT, secondary analysis).

S Single or multisite RCT.

- Number of participants.

Detail of intervention in each group.

- Type of diabetes (type 1, type 2 or gestational diabetes mellitus).

- Patient-reported outcome measure used.

- Time points during trial which the patient-reported outcomes (PROs) were measured.

- Journal of publication.

- Year of publication.

- Country of origin.

- The use of PR0 as a primary, secondary or exploratory outcome.

then be divided by the total number of available pointsfor example if a PRO is a primary outcome it must satisfy more domains than if it is a secondary outcome. We will then multiple this score by 100 (for ease of calculation) and calculate the average score for all included studies. Any individual study which scores above this number will be considered 'above average'. To facilitate a multivariate analysis to determine factors associated with 'above average' PRO reporting, data shown in box 1 will be extracted and entered into a predesigned data extraction form.

Using this method we will be able to report on the quality of reporting in a large group of RCTs and will be able to generate information on the standards of reporting in smaller subsets for example, technological and pharmacological interventions, reporting standards in type 1 versus type 2 diabetes. We will also be able to report on the frequency of reporting for each individual item on the CONSORT-PRO checklist.

During the full-text review we will compare the RCT reporting against the 25 point CONSORT statement which is 'an evidence-based, minimum set of recommendations for reporting randomised trials' ${ }^{31}$ We will use the same approach and scoring system when grading the quality of RCT reporting against the ${ }^{31}$ statement. When there is uncertainty or clarifications are required attempts will be made to contact the author of the paper for obtain further details.

To explore factors associated with higher quality or more complete reporting, we will perform a multiple regression analysis in which the dependent variables will be the CONSORT-PRO checklist score and the independent variables will be type of diabetes, type of intervention, country of study, multi-site trial, population size, primary RCT or secondary analysis of a RCT.

The quality of the individual trials will not be assessed as it is irrelevant to the aim of our systematic review. The aim of our systematic review is to establish the quality and completeness of PRO reporting in RCTs of diabetes in pregnancy. The identification and grading of these PROs 
is not based on study quality/bias and we will not be making comparisons between outcomes reported from studies at different risks of bias.

\section{Ethics and dissemination}

Ethical approval to conduct this study was obtained from the ethics committee at Galway University Hospitals on 24 March 21 (CA 2592).

We aim to publish our findings in a peer-reviewed journal and present our findings at national and international conferences.

\section{Systematic review registration}

This systematic review was registered prospectively with the International Prospective Register of Systematic Reviews (PROSPERO). Registration number CRD42021234917

\section{Patient and public involvement}

There was no patient or public involvement in the development of this systematic review protocol.

\section{DISCUSSION}

Since the early 2000s the use of PROs in experimental studies has grown exponentially. In 2009 the Food and Drug Administration (FDA) issued formal guidelines for the use and inclusion of PROs in product labelling. ${ }^{32}$ This undoubtedly contributed to the $500 \%$ increase in the inclusion of PROs in regulatory applications from 2010 to 2015. In response to this increase, in 2016 the Centre for Devices and Radiological, a branch of the FDA, moved to include PROs as evidence in their decision-making process and continue to encourage the inclusion of PROs in investigational studies. ${ }^{33}$

In Europe, the European Medicines Agency has committed to exploring 'additional methodologies to gather and use patient data from the wider patient community during benefit-risk evaluation, ${ }^{34}$

This increased emphasis on PRO data collection has without question had a knock-on effect on the inclusion of PROs in published studies. In the past 5 years there has been several publications that have highlighted the diversity of PRO measurement and the quality of PRO reporting in RCTs in areas such as cystic fibrosis, oncology and haematology. ${ }^{35-37}$

More recently, PROs have been increasingly used in diabetes. ${ }^{38}$ A joint statement by the American Diabetes Association National Institute of Diabetes and Digestive and Kidney Diseases (NIDDK) ${ }^{39}$ has highlighted the relevance of PROs in diabetes trials. A study outlining the broad range of PROMs used in type 2 diabetes has recently been published and there is much discussion regarding the most appropriate PROs to use in different types of diabetes. ${ }^{40} 41$ Their importance is also well recognised in pregnancy. ${ }^{42}$ Despite this the use of PROs in the area of diabetes in pregnancy has not been explored and to our knowledge this is the first systematic review of PROs in this field. This systematic review will summarise the PROMs currently used in diabetes in pregnancy and provide information on when and in what patient cohorts PROs are collected. It will also allow us to identify factors associated with more complete reporting. This will help clinicians and patients make informed decisions on the effectiveness of treatments.

\section{Author affiliations}

${ }^{1}$ National University of Ireland Galway College of Medicine Nursing and Health Sciences, Galway, Ireland

'Diabetes and Endocrinology, National University of Ireland Galway, Galway, Ireland

${ }^{3}$ Mayo Clinic Rochester, Rochester, Minnesota, USA

${ }^{4}$ School of Nursing and Midwifery, National University of Ireland Galway, Galway, Ireland

${ }^{5}$ HRB-Trials Methodology Research Network, National University of Ireland, Galway, Ireland

${ }^{6}$ INFANT Centre and Department of Paediatrics \& Child Health, University College Cork, Cork, Ireland

${ }^{7}$ Evidence Synthesis Ireland, National University of Ireland, Galway, Ireland

${ }^{8}$ Cochrane Ireland, National UNiversity of Ireland, Galway, Ireland

${ }^{9}$ Biochemical Medicine, Galway University Hospitals, Galway, Ireland

\section{Twitter Aoife Maria Egan @egan_am}

Contributors This study concept and design was conceived by CN, LB, DD and FD. All authors contributed to the design of inclusion and exclusion criteria. CN, FD, PMO'S, OK and AME devised the data extraction tool. CN and LD will screen titles and abstracts and following on from that will review full-text articles with support from DD, LB and DD. CN drafted the manuscript and all authors reviewed, edited and approve the final manuscript. FD is the guarantor of this review.

Funding The authors have not declared a specific grant for this research from any funding agency in the public, commercial or not-for-profit sectors.

Competing interests None declared.

Patient and public involvement Patients and/or the public were not involved in the design, or conduct, or reporting or dissemination plans of this research.

Patient consent for publication Not applicable.

Provenance and peer review Not commissioned; externally peer reviewed.

Open access This is an open access article distributed in accordance with the Creative Commons Attribution Non Commercial (CC BY-NC 4.0) license, which permits others to distribute, remix, adapt, build upon this work non-commercially, and license their derivative works on different terms, provided the original work is properly cited, appropriate credit is given, any changes made indicated, and the use is non-commercial. See: http://creativecommons.org/licenses/by-nc/4.0/.

\section{ORCID iDs}

Christine Newman http://orcid.org/0000-0003-2387-4109

Delia Bogdanet http://orcid.org/0000-0001-6127-5049

Aoife Maria Egan http://orcid.org/0000-0003-0379-9279

\section{REFERENCES}

1 Mumtaz M. Gestational diabetes mellitus. Malays J Med Sci 2000;7:4-9.

2 Yuen L, Saeedi P, Riaz M. Projections of the prevalence of hyperglycaemia in pregnancy in 2019 and beyond: results from the International diabetes federation diabetes atlas. 9th edn. Diabetes Research and Clinical Practice, 2019.

3 Weissgerber TL, Mudd LM. Preeclampsia and diabetes. Curr Diab Rep 2015;15:9.

4 Nahavandi S, Seah J-mine, Shub A, et al. Biomarkers for macrosomia prediction in pregnancies affected by diabetes. Front Endocrinol 2018;9:407.

5 Remsberg KE, McKeown RE, McFarland KF, et al. Diabetes in pregnancy and cesarean delivery. Diabetes Care 1999;22:1561-7.

6 Tinker SC, Gilboa SM, Moore CA, et al. Specific birth defects in pregnancies of women with diabetes: National birth defects prevention study, 1997-2011. Am J Obstet Gynecol 2020;222:176. e1-e11. 
7 Persson M, Norman M, Hanson U. Obstetric and perinatal outcomes in type 1 diabetic pregnancies: a large, population-based study. Diabetes Care 2009;32:2005-9.

8 Dunne FP, Avalos G, Durkan M, et al. ATLANTIC DIP: pregnancy outcome for women with pregestational diabetes along the Irish Atlantic seaboard. Diabetes Care 2009;32:1205-6.

9 Hyperglycemia and adverse pregnancy outcomes. N Engl J Med Overseas Ed 2008;358:1991-2002.

10 Sacks DA, Hadden DR, Maresh M. Frequency of gestational diabetes mellitus at collaborating centers based on IADPSG consensus panelrecommended criteria. The hyperglycemia and adverse pregnancy outcome (HAPO) study 2012;35:526-8.

11 O'Sullivan EP, Avalos G, O'Reilly M, et al. Atlantic diabetes in pregnancy (DIP): the prevalence and outcomes of gestational diabetes mellitus using new diagnostic criteria. Diabetologia 2011;54:1670-5.

12 Egan AM, Vellinga A, Harreiter J, et al. Epidemiology of gestational diabetes mellitus according to IADPSG/WHO 2013 criteria among obese pregnant women in Europe. Diabetologia 2017;60:1913-21.

13 Behboudi-Gandevani S, Amiri M, Bidhendi Yarandi R, et al. The impact of diagnostic criteria for gestational diabetes on its prevalence: a systematic review and meta-analysis. Diabetol Metab Syndr 2019;11:11.

14 O'Malley EG ODM, McArdle C, Kennedy RAK. Screening for gestational diabetes mellitus selectively in a university maternity hospital. Irish Med J 2017;771.

15 Black MH, Sacks DA, Xiang AH, et al. The relative contribution of prepregnancy overweight and obesity, gestational weight gain, and IADPSG-defined gestational diabetes mellitus to fetal overgrowth. Diabetes Care 2013;36:56-62.

16 Yogev $Y$, Xenakis EMJ, Langer $\mathrm{O}$. The association between preeclampsia and the severity of gestational diabetes: the impact of glycemic control. Am J Obstet Gynecol 2004;191:1655-60.

17 Silverman BL, Rizzo TA, Cho NH, et al. Long-term effects of the intrauterine environment. The northwestern university diabetes in pregnancy center. Diabetes Care 1998;21:B142-9.

18 O'Reilly MW, Avalos G, Dennedy MC, et al. Atlantic DIP: high prevalence of abnormal glucose tolerance post partum is reduced by breast-feeding in women with prior gestational diabetes mellitus. Eur $J$ Endocrinol 2011;165:953-9.

19 Dennedy MC, Avalos G, O'Reilly MW, et al. ATLANTIC-DIP: raised maternal body mass index (BMI) adversely affects maternal and fetal outcomes in glucose-tolerant women according to international association of diabetes and pregnancy study groups (IADPSG) criteria. J Clin Endocrinol Metab 2012;97:E608-12.

20 Simmons D. Diabetes and obesity in pregnancy. Best Pract Res Clin Obstet Gynaecol 2011;25:25-36.

21 Egede LE, Dismuke CE. Serious psychological distress and diabetes: a review of the literature. Curr Psychiatry Rep 2012;14:15-22.

22 Smith MV, Shao L, Howell H, et al. Perinatal depression and birth outcomes in a healthy start project. Matern Child Health $J$ 2011;15:401-9.

23 Schmidt CB, Voorhorst I, van de Gaar VHW, et al. Diabetes distress is associated with adverse pregnancy outcomes in women with gestational diabetes: a prospective cohort study. BMC Pregnancy Childbirth 2019;19:223.

24 Egan AM, Bogdanet D, Griffin TP, et al. A core outcome set for studies of gestational diabetes mellitus prevention and treatment. Diabetologia 2020;63:1120-7.
25 Bogdanet D, Reddin C, Macken E, et al. Follow-up at 1 year and beyond of women with gestational diabetes treated with insulin and/ or oral glucose-lowering agents: a core outcome set using a Delphi survey. Diabetologia 2019;62:2007-16.

26 Shivers JP, Mackowiak L, Anhalt H, et al. "Turn it off!": Diabetes Device Alarm Fatigue Considerations for the Present and the Future. J Diabetes Sci Technol 2013;7:789-94.

27 Johnston BC, Patrick DL, Devji T, et al. Chapter 18: patient-reported outcomes. In: Cochrane handbook for systematic reviews of interventions version 62, 2021.

28 Coronini-Cronberg S, Appleby J, Thompson J. Application of patient-reported outcome measures (PROMs) data to estimate cost-effectiveness of hernia surgery in England. J R Soc Med 2013;106:278-87.

29 Hagger V, Hendrieckx C, Cameron F, et al. Diabetes distress is more strongly associated with $\mathrm{HbA} 1 \mathrm{c}$ than depressive symptoms in adolescents with type 1 diabetes: results from diabetes miles YouthAustralia. Pediatr Diabetes 2018;19:840-7.

30 Calvert M, Blazeby J, Altman DG, et al. Reporting of patient-reported outcomes in randomized trials: the CONSORT PRO extension. JAMA 2013;309:814-22.

31 Moher D, Hopewell S, Schulz KF, et al. Consort 2010 explanation and elaboration: updated guidelines for reporting parallel group randomised trials. BMJ 2010;340:c869.

32 Administration USDoHaHSFaD. Guidance for industry patientreported outcome measures: use in medical product development to support labeling claims, 2009.

33 (CDRH) CfDaRH. centre for devices and radiological health (CDRH) 2016.

34 Sepodes B. Reinforce patient relevance in evidence generation Human Stakeholder Owrkshop, 2019.

35 Ahmed K, Kyte D, Keeley T, et al. Systematic evaluation of patientreported outcome (pro) protocol content and reporting in UK cancer clinical trials: the EPIC study protocol. BMJ Open 2016;6:e012863.

36 Chakraborty R, Cannella L, Cottone F, et al. Quality of patientreported outcome reporting in randomised controlled trials of haematological malignancies according to international quality Standards: a systematic review. Lancet Haematol 2020;7:e892-901.

37 Ratnayake I, Ahern S, Ruseckaite R. A systematic review of patientreported outcome measures (PROMs) in cystic fibrosis. BMJ Open 2020;10:e033867-e.

38 Schneider $\mathrm{D}$. The importance of patient-reported outcomes in type 2 diabetes: insight from the pioneer program with oral semaglutide. AJMC 2020;25:S356-67.

39 Marrero DG, Hilliard ME, Maahs DM, et al. Using patient reported outcomes in diabetes research and practice: recommendations from a national workshop. Diabetes Res Clin Pract 2019;153:23-9.

40 Reaney M, Elash CA, Litcher-Kelly L. Patient reported outcomes (pros) used in recent phase 3 trials for type 2 diabetes: a review of concepts assessed by these pros and factors to consider when choosing a PRO for future trials. Diabetes Res Clin Pract 2016;116:54-67.

41 Carlton J, Leaviss J, Pouwer F, et al. The suitability of patientreported outcome measures used to assess the impact of hypoglycaemia on quality of life in people with diabetes: a systematic review using COSMIN methods. Diabetologia 2021;64:1213-25.

42 Tunçalp Ö, Were WM, MacLennan C, et al. Quality of care for pregnant women and newborns-the WHO vision. BJOG 2015;122:1045-9. 